\title{
Wideband Reflector and Analogue Electromagnetically Induced Reflection in Metamaterials
}

\author{
Wei Huang ${ }^{1}$, Ningye He ${ }^{2,3}$, Renxia Ning ${ }^{2,3, *}$ and Zhenhai Chen ${ }^{2,3}$ \\ 1 State Key Laboratory of ASIC and System, Shanghai Institute of Intelligent Electronics \& Systems, \\ School of Microelectronics, Fudan University, Shanghai 200433, China; eehuangw@fudan.edu.cn \\ 2 School of Information Engineering, Huangshan University, Huangshan 245041, China; \\ hny@hsu.edu.cn (N.H.); chzh@hsu.edu.cn (Z.C.) \\ 3 Engineering Technology Research Center of Intelligent Microsystems, Huangshan 245041, China \\ * Correspondence: nrxxiner@hsu.edu.cn
}

Citation: Huang, W.; He, N.; Ning,

R.; Chen, Z. Wideband Reflector and Analogue Electromagnetically Induced Reflection in Metamaterials. Crystals 2021, 11, 985. https:// doi.org/10.3390/cryst11080985

Academic Editor: Luis

M. Garcia-Raffi

Received: 18 July 2021

Accepted: 17 August 2021

Published: 19 August 2021

Publisher's Note: MDPI stays neutral with regard to jurisdictional claims in published maps and institutional affiliations.

Copyright: (c) 2021 by the authors. Licensee MDPI, Basel, Switzerland. This article is an open access article distributed under the terms and conditions of the Creative Commons Attribution (CC BY) license (https:// creativecommons.org/licenses/by/ $4.0 /)$.
Abstract: Metamaterials are highly demanded for advanced applications in absorbers, sensors, and filters. However, metamaterial reflectors, especially broadband reflectors, remain challenging. In this paper, we theoretically investigate a wideband metamaterial reflector which consists of cross shaped graphene strips and a silica $\left(\mathrm{SiO}_{2}\right)$ substrate. The cross shaped graphene strips are coated on the top of the structure, and the cross shape rotated $45^{\circ}$ graphene strips are spun on the bottom of it. The calculated reflection can be tuned through length and width of the graphene strips. By comparison, not only broadband reflection but also analogue electromagnetically induced reflection (EIR) can be realized. Moreover, the structure can generate a bi-directional broadband reflection of insensitive polarization. This kind of bi-directional reflector at microwave frequencies is obtained because the top and bottom graphene strip structures are similar. We employ the electric field distribution of the designed structure to elucidate the mechanism of the analogue EIR effect. We further discuss the influence of incident angle on the analogue EIR effect. Such a bi-directional reflector can be potentially applied to a wideband reflector, antenna, and sensor.

Keywords: wideband reflector; analogue electromagnetically induced reflection; polarization insensitive; bi-directional

\section{Introduction}

In recent years, as a specific application direction of metamaterials, metamaterial reflectors have been applied in the gigahertz $(\mathrm{GHz})$ [1], terahertz $(\mathrm{THz})$ [2], and optical bands [3]. Especially in the microwave range, they have been widely concerned with the fields of reflective antennas [4] and polarization control [5]. The tunability of graphene materials is obvious to all. A hybrid structure of graphene/metamaterial which directly deposits a single layer of graphene on the $\mathrm{SiO}_{2} /$ silicon(Si)substrate of the ring resonator has been studied. The tunability of the structure mainly depends on the transmission band between two adjacent resonance frequencies [6]. The graphene reflector is adjustable, which makes the application more flexible [7]. Therefore, narrowband, multiband, and broadband reflectors have applications, especially broadband reflectors, which have potential applications in reflective antennas and solar equipment [8]. Most of the structures of perfect reflectors require metal reflective surfaces [7,8]. However, the bi-directional metamaterial reflector remains challenging and requires more efforts [9].

Analogue electromagnetically induced reflection (EIR) $[10,11]$, as a kind of electromagnetically induced transparency (EIT) effect, has been concerned with sensor, slow light devices [12]. A two-dimensional cavity grating structure to realize EIR was designed. The structural unit consists of an orthogonal ring resonator, a circular ring resonator, and a metal cavity. The physical mechanism of EIR is the weak hybridization between the quadrature ring resonator and the circular ring resonator between $15.15 \mathrm{GHz}$, showing a 
high reflection coefficient of 95\% [13]. A dynamically adjustable electromagnetic induction reflection structure based on complementary graphene metamaterials is composed of a wire slot and a split ring resonator slot array structure. In this structure, the dark mode excited by the near-field coupling between the wire slot and the split ring resonator slot structure creates a reflection window [10]. A graphene-based metamaterial with a dynamically adjustable terahertz EIR window was studied. The metamaterial includes a single layer of graphene with an array of wire slot pairs and a split ring resonator slot structure printed on a $\mathrm{SiO}_{2} / \mathrm{Si}$ substrate. The wire slot pair and the resonant ring slot structure can function as super-radiation and sub-radiation elements [14].

A parallel double stub resonator shoulder coupling two metal-insulator-metal waveguides is proposed. The transmission characteristics of the plasma-induced reflection effect are completely opposite to the transmission characteristics of the plasma-induced transparent system [15]. The EIR effect was studied in the microwave [13], THz [16], and mid-infrared region [17]. A dual-mode controllable terahertz metasurface based on a complementary plasmon-induced reflection structure was designed. Both photosensitive silicon and single-layer graphene are integrated into the unit cell, and through optical pumping and additional voltage modulation, respectively, the active control of the EITlike reflection window and the entire resonance is realized [18]. To sum up, bi-directional broadband reflectors and EIR devices have potential applications in reflective antennas, reflective devices, and reflective sensors.

In this paper, a graphene metamaterial tri-layer structure was theoretically designed. The top and bottom layers are graphene strips with the analogous structure. The bottom layer structure is rotated at an angle $\varphi$ in the $z$ direction. The reflection of the structure is obtained by the finite difference time domain method. The calculated results show that the structure is in the low frequency range and has a broadband bi-direction reflector, and the high frequency band has an analogue EIR effect. The physical mechanism of analogue EIR is analyzed through the electric field distribution. The change of the reflection window is studied by changing the incident angle and the dielectric constant of the environment. Because the structure has rotational symmetry in the $x$ and $y$ directions, the structure also has the analogue EIR effect of polarization insensitivity. The above research results have potential applications in reflective antennas, large-angle reflectors, and refractive index sensors.

\section{Model and Simulation}

The three-layer metamaterial structure designed is shown in Figure 1. The substrate layer is $\mathrm{SiO}_{2}$, and the top and bottom layers are graphene layers of the analogue shape, composed of the graphene strips rotated $45^{\circ}$ to each other. The length and width of the graphene strip are $l_{\mathrm{s}}$ and $w_{\mathrm{s}}$. The graphene layer on the bottom and top layers differs by an angle $\varphi$ on the $z$-axis. In this structure, metal materials such as gold and copper can also be selected for the graphene layer, but metal loss will occur. The $\mathrm{SiO}_{2}$ substrate layer size is $l \times l$, the thickness is $t_{2}$, and the thickness of the bottom and top graphene strips is $t_{1}$. The dielectric constant with $\mathrm{SiO}_{2}$ is 3.78. We used the finite integral time domain (FITD) method, where $x, y$ is the unit cell boundary condition, and in the $z$ direction is the opening (add space).

To analyze the response of the structure to electromagnetic waves, the parameters of the structure are: $t_{1}=1 \mathrm{~nm}, t_{2}=0.5 \mathrm{~mm}, l=10 \mathrm{~mm}, l_{\mathrm{s}}=8.5 \mathrm{~mm}, w_{\mathrm{s}}=0.5 \mathrm{~mm}, \varphi=22.5^{\circ}$.

The conductivity of graphene $\sigma_{\mathrm{G}}$ was shown in the Kubo model as Equation (1) [19].

$$
\sigma_{G}=\sigma_{\text {inter }}+\sigma_{\text {intra }}
$$

Here, $\sigma_{\text {inter }}$ is taken into account as inter-band and $\sigma_{\text {intra }}$ is intra-band conductivity. 

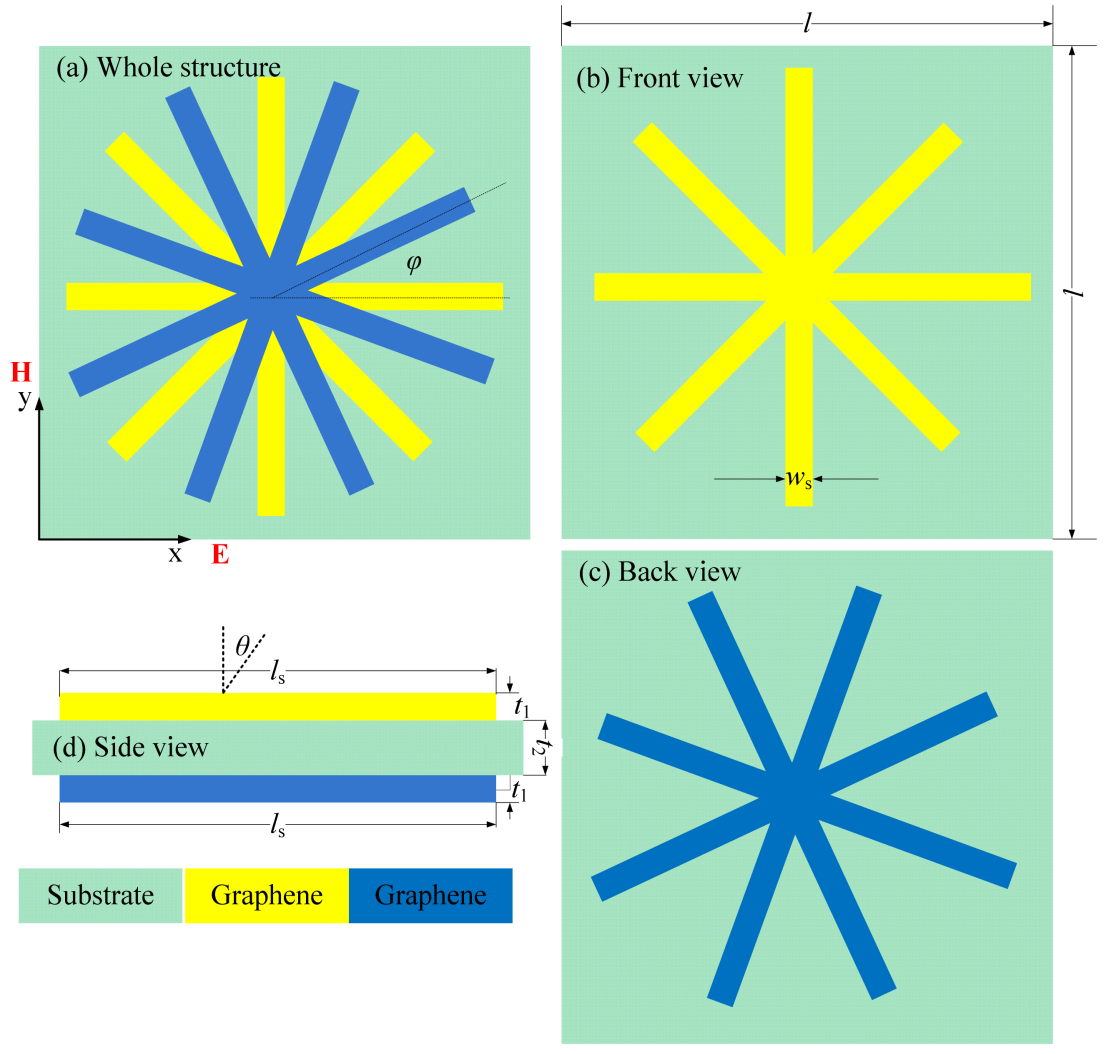

Figure 1. Unit cell of the bi-direction graphene metasurface. (a) Unit cell of the whole structure. (b) Front view of the structure. (c) Back view of the structure. (d) Side view of the structure.

The inter-band conductivity $\sigma_{\text {inter }}$ is given by

$$
\sigma_{\text {inter }}=\frac{i e^{2} k_{B} T}{\pi \hbar^{2}\left(\omega+\frac{i}{\tau}\right)}\left(\frac{\mu_{c}}{k_{B} T}+2 \ln \left(e^{-\frac{\mu_{c}}{k_{B} T}}+1\right)\right.
$$

The intra-band conductivity $\sigma_{\text {intra }}$ is given by

$$
\sigma=\frac{i e^{2}}{4 \pi \hbar} \ln \left|\frac{2 \mu_{c}-\hbar\left(\omega+\frac{i}{\tau}\right)}{2 \mu_{c}+\hbar\left(\omega+\frac{i}{\tau}\right)}\right|
$$

where $\omega$ is radian frequency, $\hbar$ is the reduced Planck constant, $\kappa_{\mathrm{B}}$ is the Boltzman constant, $e$ is the charge of an electron, $T$ is the temperature, $\mu_{\mathrm{c}}$ denotes the chemical potential, and $\tau$ is electron-phonon relaxation time, respectively.

Assuming that the electronic band structure of a graphene sheet is not affected by the neighbor, the effective permittivity $\varepsilon_{G}$ of the graphene can be written as [20]

$$
\varepsilon_{G}=1+\sigma_{G} / t_{1} \omega \varepsilon_{0}
$$

where, $t_{1}$ is the thickness of the graphene sheet, and $\varepsilon_{0}$ is the permittivity in the vacuum.

Figure 2 shows that variation of the real part and imaginary part of the effective permittivity of the graphene with the $\mu_{\mathrm{c}}$ at $\tau=1 \mathrm{fs}$. The real part of $\varepsilon_{\mathrm{G}}$ decreases with the $\mu_{c}$, and the increase remains almost constant in Figure 2a. Additionally, the imaginary part increases with the increase of $\mu_{c}$ and decreases with the increase of frequency. 


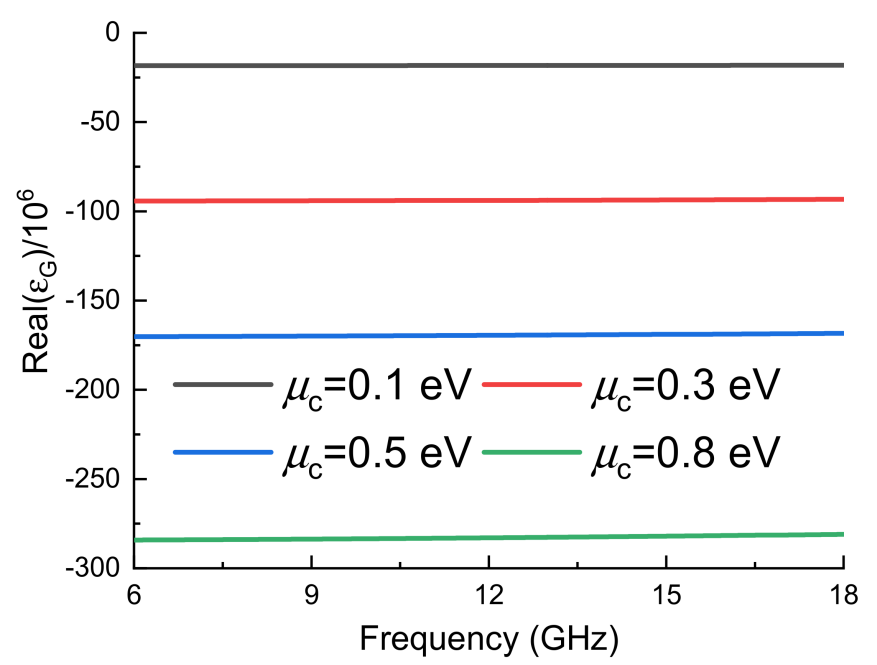

(a)

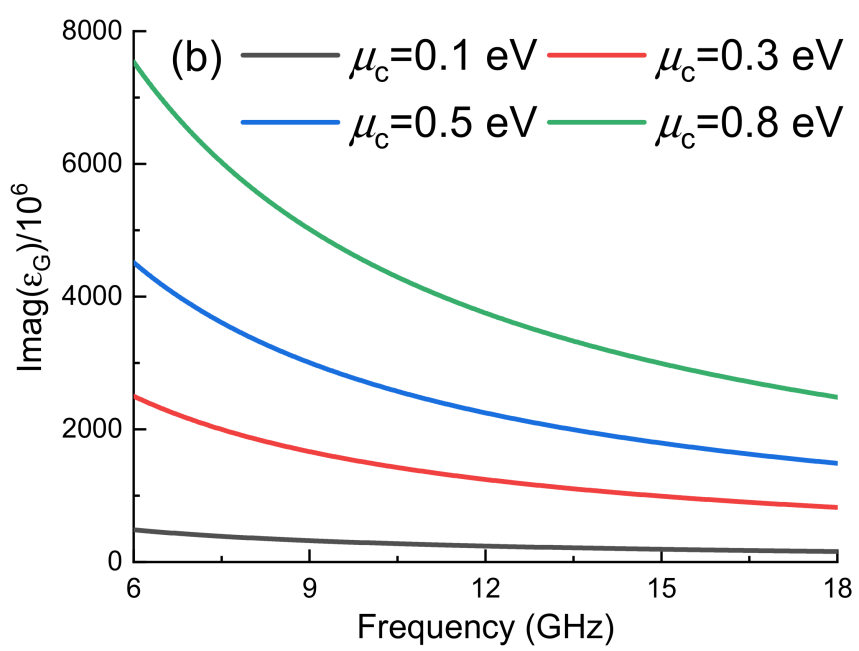

(b)

Figure 2. (a) Real part and (b) imaginary part of the effective permittivity of graphene for $\mu_{\mathrm{c}}$ of 0.1 , $0.3,0.5$, and $0.8 \mathrm{eV}$.

Figure 3 shows the reflection spectra of the structure in the $+z$ and $-z$ directions in transverse electric (TE) and transverse magnetic (TM) polarized waves. It is demonstrated that the structure has the wideband reflection of polarization insensitivity, and the bidirectional reflector can be obtained. As a result of the structure having symmetry on the $x$ and $y$ axes, it is insensitive to TE and TM mode polarization. In addition, due to the consistency of the bottom and top layer in the structure, regardless of whether the incident electromagnetic wave is input from the $+z$ or $-z$ direction, there is almost no change in reflection spectra.

Figure 4 shows the reflectance spectrum of the partial structure and whole structure. To further explore the result, the structure was split into Structure I containing the substrate and the top graphene layer and Structure II containing the substrate and the bottom graphene layer, and the graphene strips in Structure I and Structure II constitute the complete sandwich structure. By comparison, it can be found that the complete structure produces a broadband reflection spectrum in the range of $8.58-13 \mathrm{GHz}$ (the bandwidth (BW) is $4.42 \mathrm{GHz}$ ), with a reflection exceeding 0.9 , and a narrowband analogue EIR window around $13 \mathrm{GHz}$ was found. 


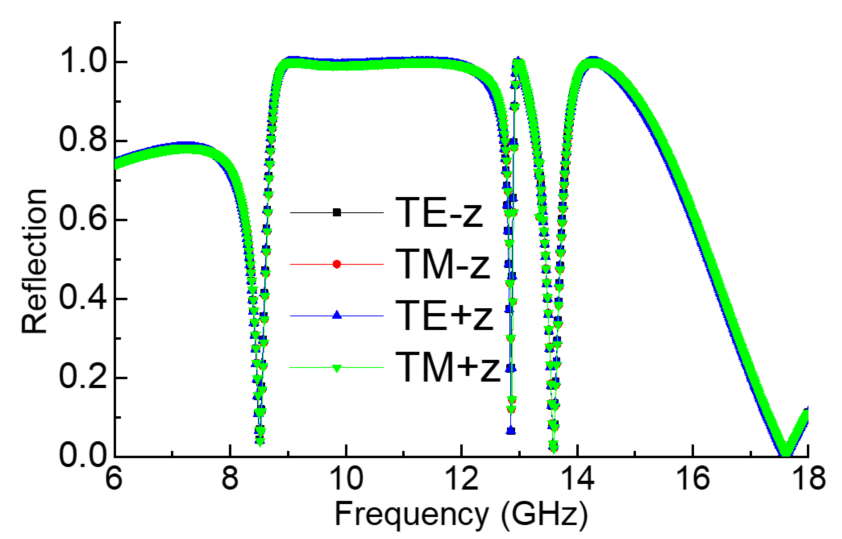

Figure 3. Reflection spectra of the tri-layers structure from TE, TM mode, $+z$ and $-z$ direction, respectively.

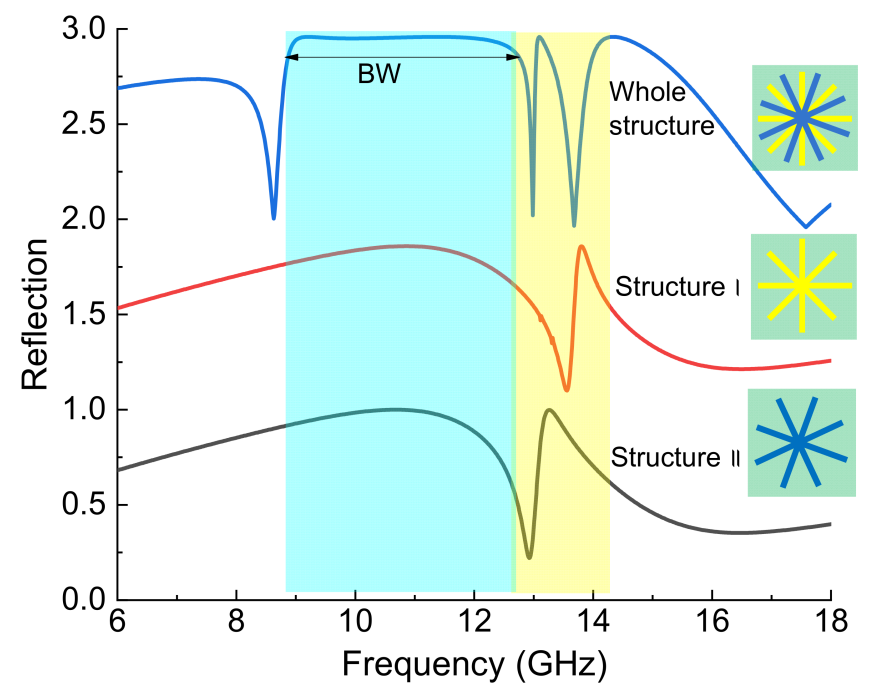

Figure 4. Reflection spectra comparison of fractional structures.

To further discuss the reflection spectrum on the high frequency band of the structure, Figure 5 shows the reflection spectra between $12 \mathrm{GHz}$ and $15 \mathrm{GHz}$. It can be seen that Structure I resonates around 13.5 GHz, and the resonance frequency of Structure II produces a red shift; the frequency is about $12.8 \mathrm{GHz}$. The reflection spectrum of the complete structure can be obtained, and the structure produces an analogue EIR effect. The interesting result here is that by rotating the angle of the graphene strips, an analogue EIR phenomenon is easily observed.

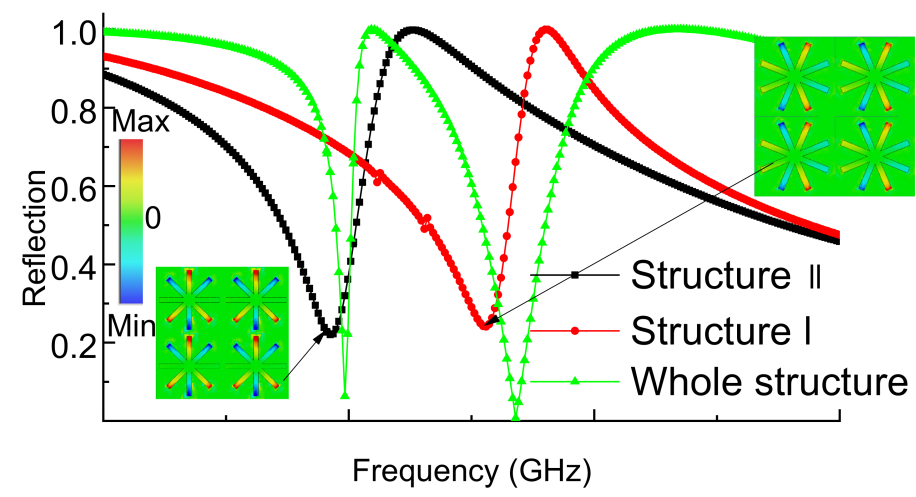

Figure 5. Reflection spectra of high frequency range. 
Figure 6 shows the electric field distribution of the complete structure. Figure $6 \mathrm{a}$ shows the electric field distribution at the resonant frequency of $8.58 \mathrm{GHz}$. The electric field distribution is mainly concentrated in the structure in the $y$ direction. It is demonstrated that the structure has fundamental resonance to an electromagnetic wave. To further study the mechanism of the analogue EIR effect, from Figure $6 \mathrm{~b}$, it can be seen that at the frequency of $12.984 \mathrm{GHz}$, the electric field energy is mainly concentrated on the graphene strip on the top layer of the structure. Compared with Figure 6a, it can be seen that the electric field distribution on the graphene strip on the diagonal of the top layer of the structure is opposite to that in Figure 6a. Figure 6c shows that the electric field energy of $13.104 \mathrm{GHz}$ is mainly concentrated on the graphene strips on the top layer and the graphene strips on the bottom layer, indicating that the electric field energy is coupled from the top graphene layer as a bright mode to the bottom graphene layer as a dark mode. Moreover, the direction of the induced current is opposite to that in Figure 6c. It can be seen from Figure $6 \mathrm{~d}$ that at the frequency of $13.692 \mathrm{GHz}$, the electric field energy of the top layer is weak and is mainly concentrated at the intersection of the graphene strips on the bottom layer, demonstrating that the energy has been coupled from the top layer to the bottom layer, thereby generating the analogue EIR effect [21-23].

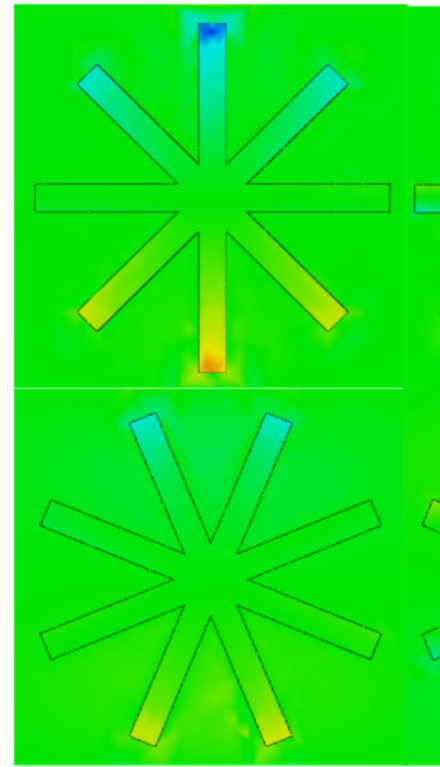

(a) $8.58 \mathrm{GHz}$
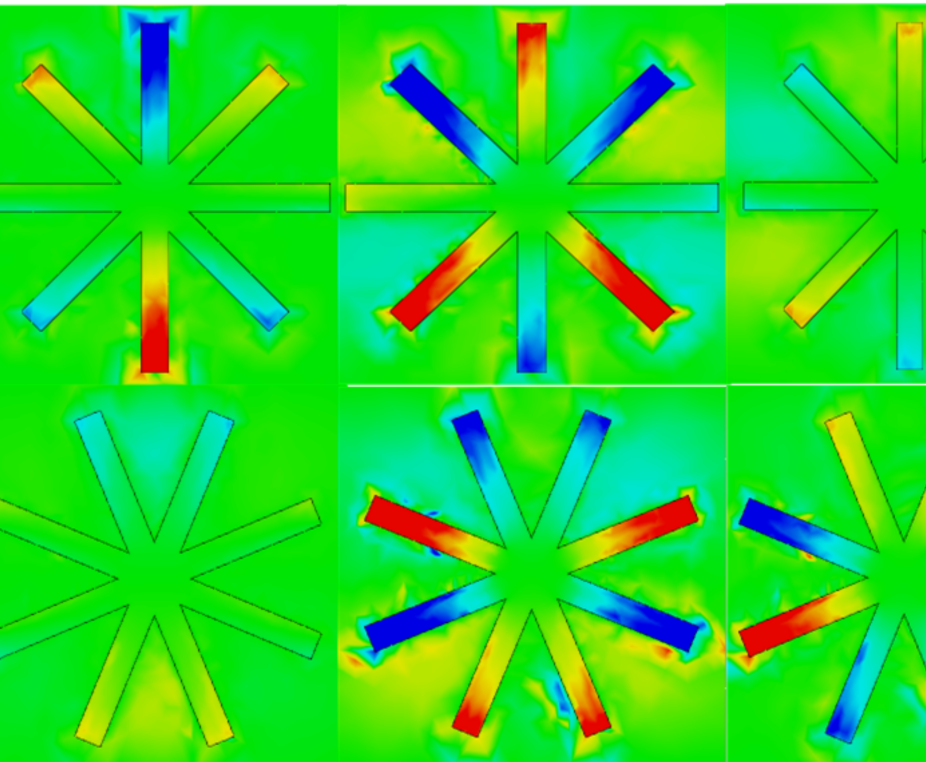

(b) $12.984 \mathrm{GHz}$ (c) $13.104 \mathrm{GHz}$

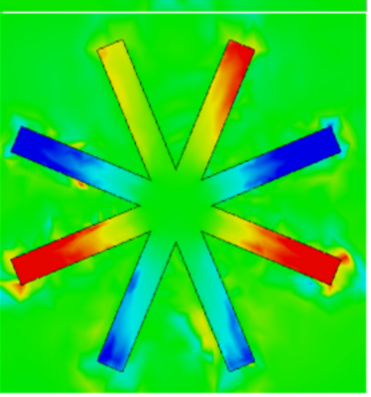

(d) $13.692 \mathrm{GHz}$

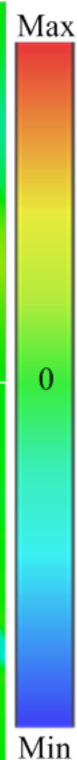

Min

Figure 6. The electric field distribution of the (a) $8.58 \mathrm{GHz}$, (b) $12.984 \mathrm{GHz}$, (c) $13.104 \mathrm{GHz}$, and (d) $13.692 \mathrm{GHz}$.

\section{Results and Discussion}

To further study the wideband reflection of the structure, Figure 7 shows the change of the reflection spectrum at the rotation angle $\varphi$ of the underlying graphene strips changing from $0^{\circ}$ to $22.5^{\circ}$. It can be seen from Figure 7 that the low frequency resonant frequency has a small variation. However, when it is larger than $10^{\circ}$, a new resonant frequency of about $13 \mathrm{GHz}$ results in $\varphi$ changing from 10 to $22.5^{\circ}$. When the graphene strips at the top and bottom of the structure produce a certain phase offset, a new resonance will occur. The phenomenon that produces the analogue EIR effect was shown in Figure 5. Additionally, the bandwidth of the wideband reflector decreases slightly.

Figure 8 shows the effect of the graphene strip length $l_{\mathrm{S}}$ on the reflection window. It can be seen that increasing the $l_{1}$ produces a significant red shift of the reflection window, and the bandwidth of the reflection window is reduced to a certain extent. It can be seen from the illustration of electric field distribution in Figure 5 that the resonance frequencies 
of Structures I and II are affected by the length of the graphene strip. Therefore, the above results are produced.

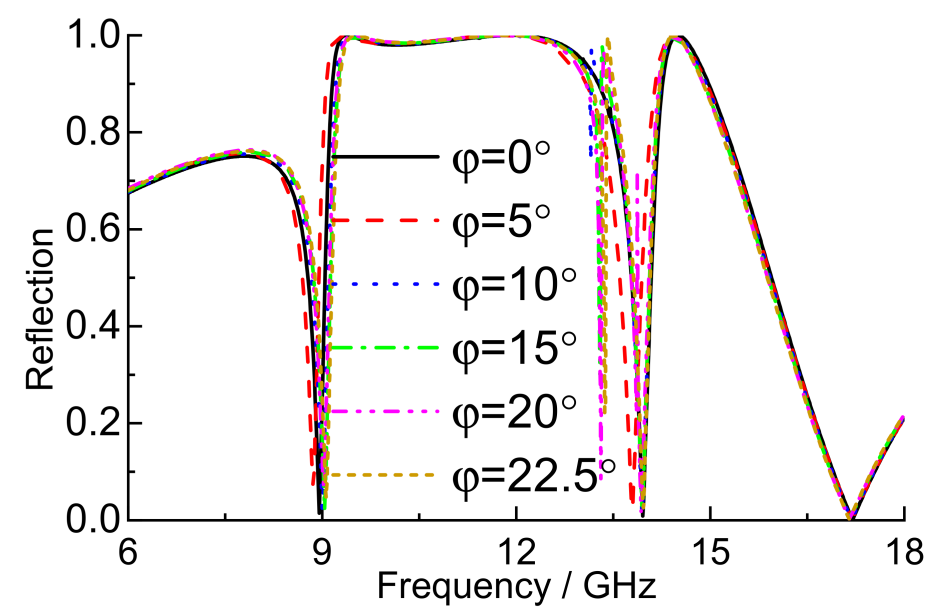

Figure 7. Comparison of the reflection of the top and bottom graphene strip with phase $\varphi$.

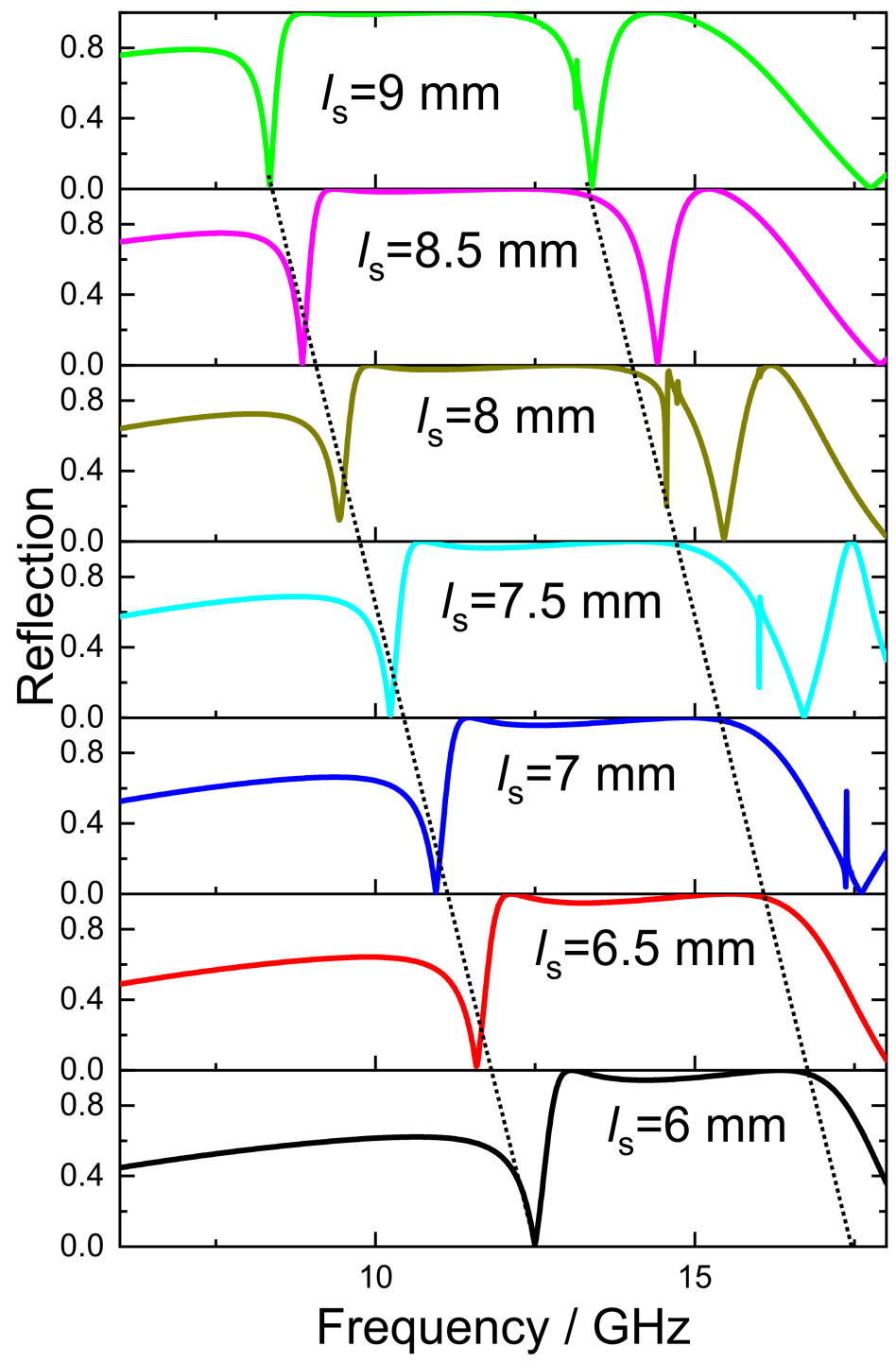

Figure 8. The effect of changing the length of graphene strip $l_{\mathrm{S}}$ on the reflection window. 
In the next stage, we study the reflection spectra for different widths of the graphene strip $w_{\mathrm{s}}$. The width of $w_{\mathrm{s}}$ is reduced to be $0.2 \mathrm{~mm}$ while keeping the length of $l_{\mathrm{s}}$ fixed at $8.5 \mathrm{~mm}$. For this asymmetric structure, as can be seen in Figure 9, the bandwidth of the reflection window is obviously reduced.

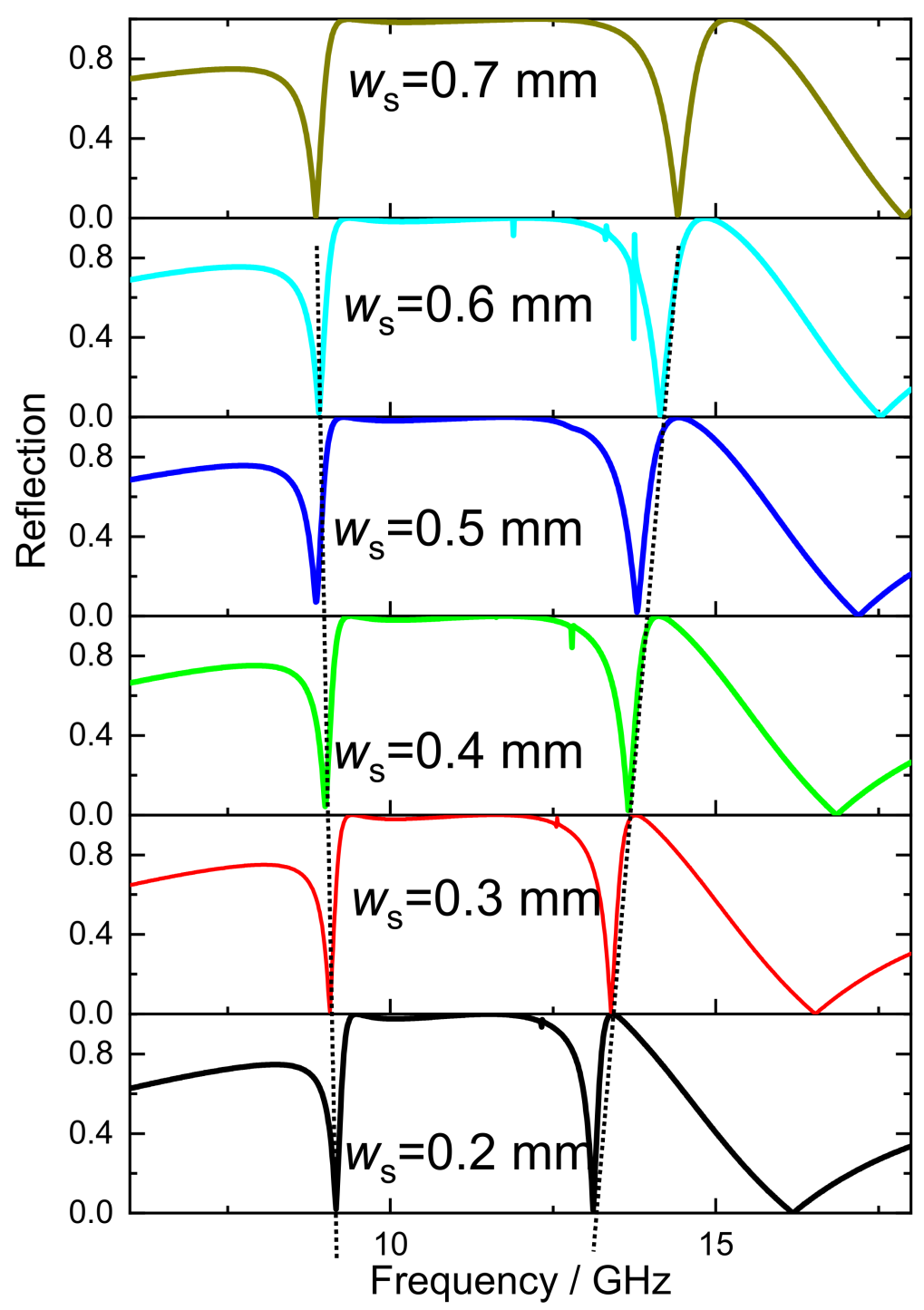

Figure 9. The effect of changing the width of graphene strip $w_{\mathrm{s}}$ on the reflection window.

Figure 10 shows the effect of changing the angle $\theta$ of incident electromagnetic waves on the reflectivity of the structure. From the reflection spectrum, it can be found that when the angle $\theta$ increases, the resonance of the middle and high frequency bands will have a greater influence, which will produce an obvious red shift, while the resonance of the low frequency is almost unchanged at around $8.5 \mathrm{GHz}$. The new resonant frequencies will be generated by oblique incidence, because at oblique incidence, the incident wave will also produce new components on the $x$ and $y$ axes. Therefore, when $\theta$ is increased to $80^{\circ}$, there is still a reflection bandwidth of about $2 \mathrm{GHz}$. 


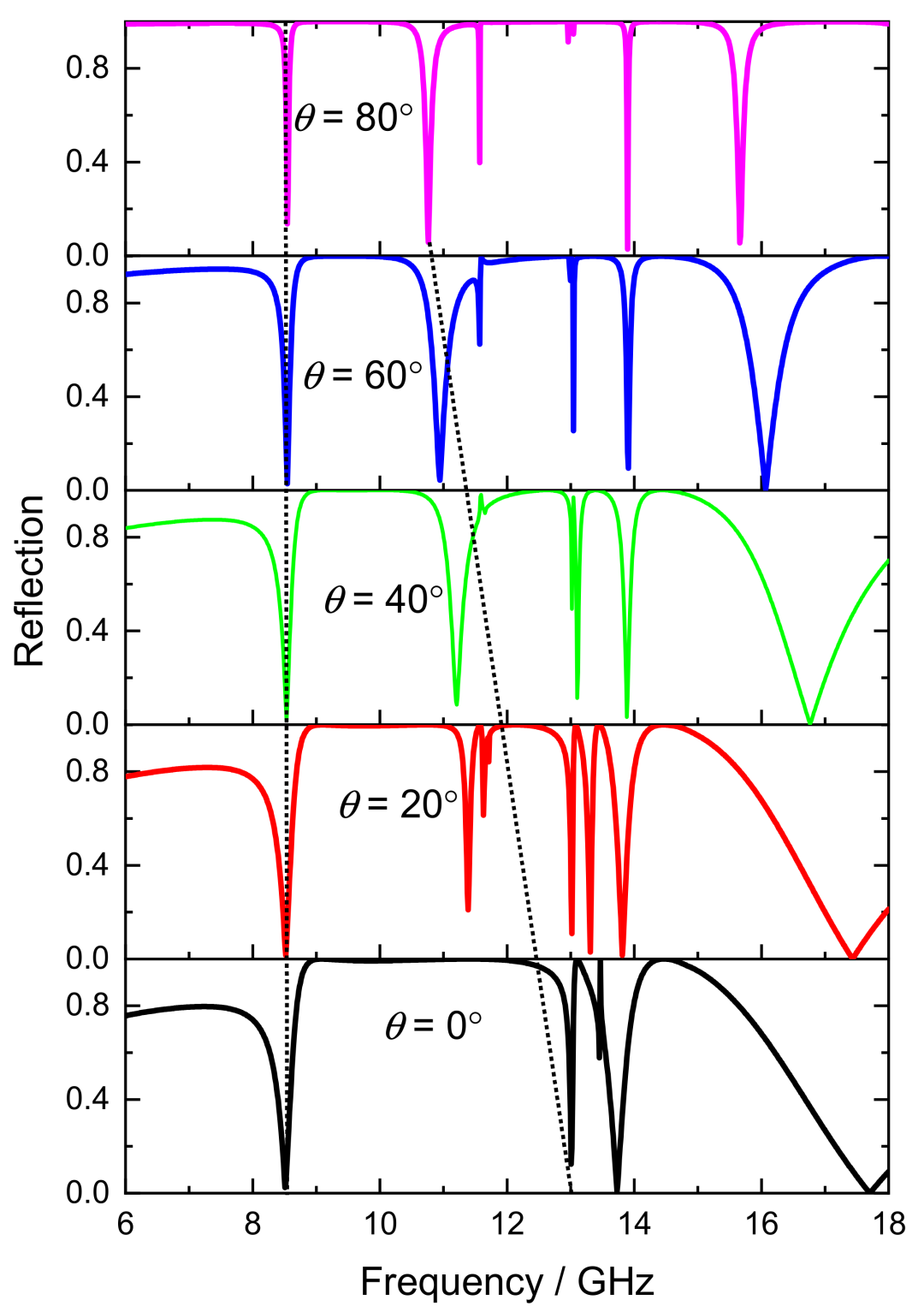

Figure 10. Variation reflection of the structure with incident angle $\theta$.

Figure 11a shows the effect of changing the environmental permittivity $\varepsilon_{\mathrm{b}}$ on the reflection. Increasing the permittivity $\varepsilon_{\mathrm{b}}$ will cause a red shift in the reflection window, and the offset of the resonance frequency in the low frequency range is slightly smaller than that of the mid and high frequency. This result showed that the designed structure can be used as a refractive index sensor [24,25]. The sensitivity $(S)$ is an important index of a refractive index sensor, and it can be expressed by $\Delta f / \Delta n$ [26]. As shown in Figure 11b, there is a larger $S$ on the low frequency $\left(f_{1}\right)$ and a smaller $S$ on the high frequencies $\left(f_{2}\right.$ and $\left.f_{3}\right)$. 


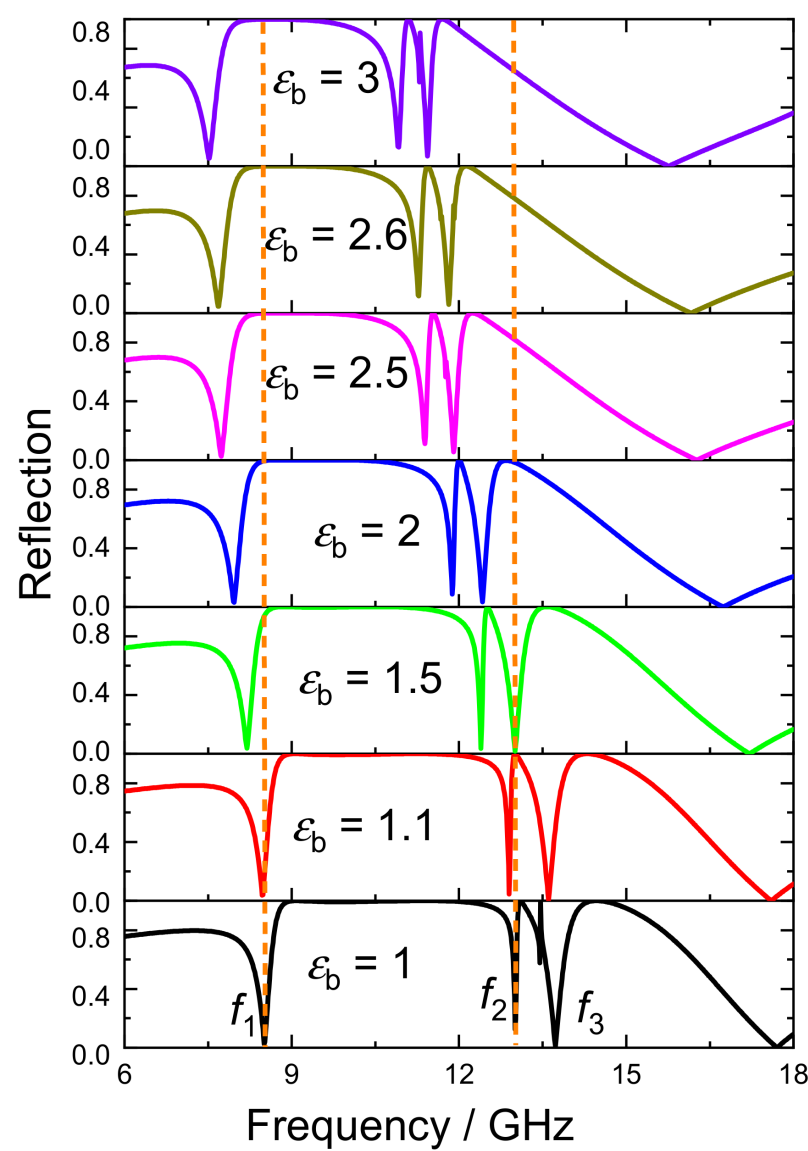

(a)

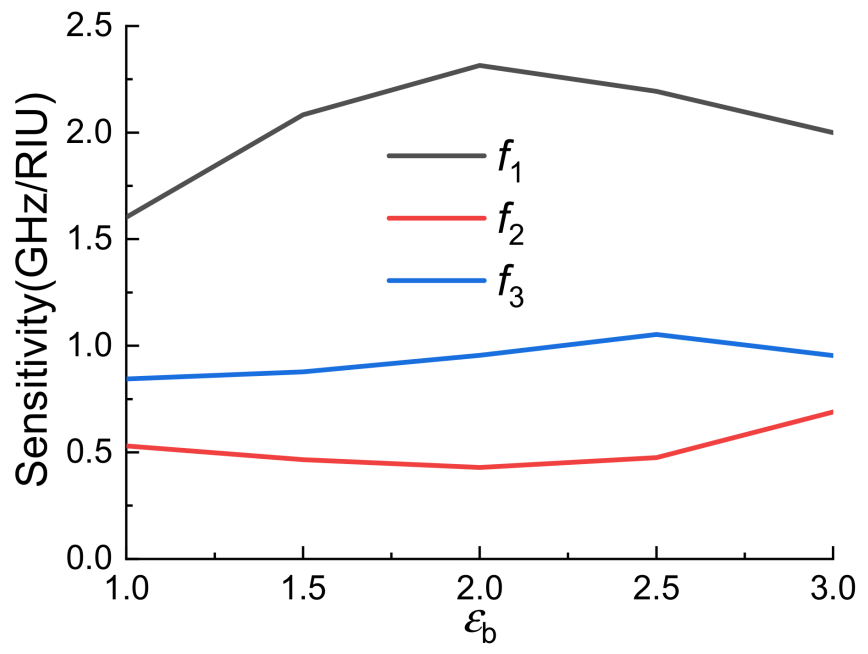

(b)

Figure 11. (a) Reflection spectrum with different permittivity of the surrounding environment $\varepsilon_{\mathbf{b}}$. (b) Sensitivity of the reflector as refractive index sensor.

\section{Conclusions}

In this paper, a graphene metamaterial with a three-layer structure was investigated. The bottom and top graphene strips have the same size and a phase shift $\varphi$ in the $z$ direction. The reflection window of the structure was analyzed. The results show that the structure has a broadband reflection in the low frequency range, and an analogue EIR effect in the 
high frequency range. The electric field distribution is further analyzed to exhibit the mechanism of the analogue EIR effect. The influence of the structure on the reflection is discussed through the change of the graphene strips. The influence of the incident electromagnetic wave angle and environmental permittivity on the reflection window is also studied. Because the structure has rotational symmetry, the structure also has polarization insensitivity and a bi-directional wideband reflection. The above research results have potential applications in bi-directional wideband reflectors, slow light devices, and refractive index sensors.

Author Contributions: Conceptualization, W.H. and R.N.; methodology, R.N.; software, N.H.; validation, W.H., N.H. and R.N.; software, R.N.; validation, Z.C., N.H. and W.H.; formal analysis, R.N.; investigation, W.H.; resources, Z.C.; data curation, N.H.; writing—original draft preparation, R.N.; writing-review and editing, W.H.; funding acquisition, R.N. All authors have read and agreed to the published version of the manuscript.

Funding: This research was funded by Natural Science Research Project of Anhui Province Education Department, grant number KJHS2020B07, KJ2020A0684.

Institutional Review Board Statement: Not applicable.

Informed Consent Statement: Not applicable.

Data Availability Statement: The data will be made immediately available based on the request.

Conflicts of Interest: The authors declare that we have no conflict of interest.

\section{References}

1. So, H.; Ando, A.; Seki, T.; Kawashima, M.; Sugiyama, T. Multiband Sector Antenna with the Same Beamwidth Employing Multiple Woodpile Metamaterial Reflectors. IEICE Trans. Electron. 2014, E97.C, 976-985. [CrossRef]

2. Jiang, L.; Fang, B.; Yan, Z.; Li, C.; Gan, H.; He, Y.; Hong, Z.; Jing, X. Broadband and wide angle perfect reflection by super periodic cubes metamaterials in terahertz region. Microelectron. Eng. 2020, 223, 111216. [CrossRef]

3. Qiu, J.; Liu, X.; Liang, Z.; Zhu, J. Ultra-wideband perfect reflection and tunneling by all-dielectric metamaterials. Opt. Lett. 2021, 46, 849-852. [CrossRef] [PubMed]

4. Guo, W.L.; Chen, K.; Wang, G.M.; Luo, X.Y.; Feng, Y.J.; Qiu, C.W. Transmission-Reflection-Selective Metasurface and Its Application to RCS Reduction of High-Gain Reflector Antenna. IEEE Trans. Antennas Propag. 2020, 68, 1426. [CrossRef]

5. Zhang, Z.; Cao, X.; Gao, J.; Li, S. Broadband Metamaterial Reflectors for Polarization Manipulation Based on Cross/Ring Resonators. Radioengineering 2016, 25, 436-441. [CrossRef]

6. Xu, H.; Chen, Z.; He, Z.; Nie, G.; Li, D. Terahertz tunable optical dual-functional slow light reflector based on gold-graphene metamaterials. New J. Phys. 2020, 22, 123009. [CrossRef]

7. Yuan, C.; Yang, R.; Wang, J.; Tian, J. Tian Tunable enhanced bandwidth all-graphene -dielectric terahertz metamaterial absorber/reflector. Optik 2020, 224, 165517. [CrossRef]

8. Deng, G.; Xia, T.; Yang, J.; Qiu, L.; Yin, Z. Qiu and Z. Yin Tunable terahertz metamaterial with a graphene reflector. Mater. Res. Express 2016, 3, 115801. [CrossRef]

9. Ma, Y.; Li, D.; Chen, Z.; Qian, H.; Ning, R. Multiband reciprocal polarization insensitivity electromagnetically induced transparency in metasurfaces. J. Opt. 2020, 22, 055101. [CrossRef]

10. Jiang, J.; Zhang, Q.; Ma, Q.; Yan, S.; Wu, F.; He, X. Dynamically tunable electromagnetically induced reflection in terahertz complementary graphene metamaterials. Opt. Mater. Express 2015, 5, 1962-1971. [CrossRef]

11. Liu, Y.; Zhang, Y.Q.; Jin, X.R.; Zhang, S.; Lee, Y.P. Dual-band infrared perfect absorber for plasmonic sensor based on the electromagnetically induced reflection-like effect. Opt. Commun. 2016, 371, 173-177. [CrossRef]

12. Zhao, X.; Yi, J.; Burokur, S.N. Direct dark mode excitation of electromagnetically induced reflection effect in enantiomer-based metasurface and its application in terahertz detection. In Proceedings of the IEEE International Symposium on Antennas and Propagation and North American Radio Science Meeting, Montreal, QC, Canada, 5-10 July 2020.

13. Shen, Z.; Yang, D.; Xia, Y.; Huang, X. Metamaterial-inspired 2D cavity grating with electromagnetically induced reflection as a glucose sensor. Phys. Scr. 2020, 96, 025502. [CrossRef]

14. He, X.; Yao, Y.; Huang, Y.; Zhang, Q.; Zhu, L.; Wu, F.; Ying, G.; Jiang, J. Active manipulation of electromagnetically induced reflection in complementary terahertz graphene metamaterial. Opt. Commun. 2018, 407, 386-391. [CrossRef]

15. Liu, B.; Liao, Y.C.; Hu, J.F.; Liu, J.; He, X.D.; Chen, Z.P. Plasmon-induced reflection and its application for all-optical diode based on paralleled double-stub resonators. Appl. Phys. Express 2019, 12, 032011. [CrossRef]

16. Noual, A.; Amrani, M.; El Boudouti, E.H.; Pennec, Y.; Djafari-Rouhani, B. Terahertz multi-plasmon induced reflection and transmission and sensor devices in a graphene-based coupled nanoribbons resonators. Opt. Commun. 2019, 440, 1-13. [CrossRef] 
17. Janfaza, M.; Mansouri-Birjandi, M.A.; Tavousi, A. Tunable plasmon-induced reflection based on graphene nanoribbon Fabry-Perot resonator and nanodisks. Opt. Mater. 2018, 84, 675-680. [CrossRef]

18. Yang, Y.; Li, J.; Li, J.; Huang, J.; Zhang, Y.; Liang, L.; Yao, J. Plasmon-induced reflection metasurface with dual-mode modulation for multi-functional THz devices. Opt. Lasers Eng. 2020, 127, 105969. [CrossRef]

19. Falkovsky, L.A. Optical properties of graphene. J. Phys. Conf. Ser. 2008, 129, 012004. [CrossRef]

20. Mikhailov, S.A.; Ziegler, K. New electromagnetic mode in graphene. Phys. Rev. Lett. 2007, 99, 016803. [CrossRef]

21. Zhu, L.; Dong, L.; Guo, J.; Meng, F.Y.; Wu, Q. Tunable electromagnetically induced transparency in hybrid graphene/all-dielectric metamaterial. Appl. Phys. A 2017, 123, 192. [CrossRef]

22. Yang, D.; Shen, Z.; Xia, Y. Design of a bi-functional metamaterial with broadband electromagnetically induced transparency and transmission-type polarization conversion. Appl. Phys. B 2021, 127, 87. [CrossRef]

23. Chu, Q.; Song, Z.; Liu, Q.H. Omnidirectional tunable terahertz analog of electromagnetically induced transparency realized by isotropic vanadium dioxide metasurfaces. Appl. Phys. Express 2018, 11, 082203. [CrossRef]

24. Jia, Z.; Huang, L.; Su, J.; Tang, B. Tunable Electromagnetically Induced Transparency-Like in Graphene metasurfaces and its Application as a Refractive Index Sensor. J. Lightwave Technol. 2021, 395, 1544. [CrossRef]

25. Sarkar, R.; Devi, K.M.; Ghindani, D.; Prabhu, S.S.; Chowdhury, D.R.; Kumar, G. Polarization independent double-band electromagnetically induced transparency effect in terahertz metamaterials. J. Opt. 2020, 22, 035105. [CrossRef]

26. Sun, R.; Li, W.; Meng, T.; Zhao, G. Design and optimization of terahertz metamaterial sensor with high sensing performance. Opt. Commun. 2021, 494, 127051. [CrossRef] 